\title{
Purging inbreeding depression and the probability of extinction: full-sib mating
}

\author{
PHILIP W. HEDRICK \\ Department of Zoology, Arizona State University, Tempe, Arizona 85287, U.S.A.
}

\begin{abstract}
Inbreeding depression has been a topic of interest in recent years from a number of perspectives, particularly in the captive breeding of endangered species. Generally, the goal of captive breeding is to avoid the detrimental effects of inbreeding depression and to retain genetic variation for future adaptation. However, an important component of apother suggested approach to captive breeding is to purge rapidly the population of its genetic load so that its long-term fitness is not compromised. I have examined the effectiveness of purging the genetic load by documenting both the reduction in inbreeding depression and the increase of the probability of extinction when there is continous fullsib mating. When the genetic load is the result of lethals, the inbreeding depression is quickly purged without a high probability of extinction, except when the total genetic load is high. On the other hand, if the load is due to detrimentals of relatively small effect, the genetic load becomes fixed, the mean fitness is reduced, and the probability of extinction may be greatly increased. In other words, the success of such a programme to purge genetic load without an increase in the probability of extinction is highly dependent upon the genetic basis of inbreeding depression, information that is not readily available for most species.
\end{abstract}

Keywords: captive breeding, full-sib mating, genetic load, inbreeding depression, lethals.

\section{Introduction}

The detrimental effects of inbreeding have been long known and were even studied and discussed by Darwin $(1868,1876)$. Early in the development of Mendelian genetics, it was understood that the increased homozygosity resulting from inbreeding caused a loss in fitness, a phenomenon termed inbreeding depression (see Lerner, 1954; Wright, 1977; Charlesworth \& Charlesworth, 1987, for extensive discussions). Although the joint effects of inbreeding and selection have been investigated for many decades (Haldane, 1937; Reeve, 1955; Brown, 1979; Charlesworth \& Charlesworth, 1987; Hedrick, 1990), the consequences of the simultaneous operation of these two evolutionary factors in a population are still not completely understood. These depend not only upon the complex manner in which both inbreeding and selection can occur but also on the variety of situations in which this combination of factors might be of evolutionary significance. Furthermore, the joint effects of inbreeding and selection are poorly understood when populations become extinct as a result of these forces. This complicated situation is particularly important for small captive populations of endangered species which may be undergoing inbreeding but for which the avoidance of extinction is of fundamental significance.

The general genetic goals of captive breeding of endangered species are to avoid inbreeding depression and to maintain genetic variation (Hedrick \& Miller, 1992). In an effort to provide the basis for the size of a captive population which would not experience detrimental inbreeding effects on fitness, Franklin (1980) suggested as guidance that 'animal breeders accept inbreeding coefficients as high as a one per cent increase per generation (i.e. an effective population size, $N_{\mathrm{e}}$, of 50) in domestic animals without great concern'. The logic for this proposal is that relatively slow inbreeding allows selection to remove deleterious alleles without greatly reducing the fitness of the population.

However, in discussing their management of Speke's gazelle, Templeton \& Read (1984) suggested that 'inbreeding depression can be rapidly and effectively reduced by an appropriate breeding program.' Their approach has been interpreted to mean that deliberate inbreeding may be used to purge a population of lethals or other genes causing inbreeding depression. Related to this approach, it has also been suggested that natural populations may become 'adapted' to inbreeding by the 
selective elimination of the detrimental and lethal alleles responsible for inbreeding depression (Templeton \& Read, 1983; Lacy, 1992). For example, after a period in which there was a very small population size or substantial inbreeding, there might be very few lethals or detrimentals left in the population so that further inbreeding would not result in a further reduction in fitness. In this regard, population genetics theory predicts that the frequency of recessive lethals would be lower in a population that has had a low population size in the past than in a larger population (Lande \& Barrowclough, 1987). If these suggestions are true, then purging inbreeding depression may be an important aspect of a captive breeding plan because one of the goals of captive breeding, that is, avoiding the reduction of population size due to inbreeding depression, may be fulfilled.

However, there are several possible negative consequences of this approach which point out the special concerns of inbreeding and selection in endangered species. Because there is generally only one 'population' of a species left when a captive breeding programme is initiated, the overriding concern is avoiding extinction of that population. Therefore, a major emphasis of this study is not just the rate of purging, but the increase in the risk of extinction during such a programme. During the actual process of purging inbreeding depression, increased homozygosity may reduce the population fitness and, as a result, increase the probability of extinction. Further, during the process of attempted purging, detrimental alleles may become fixed, thereby permanently lowering the fitness of the population and increasing the long-term probability of extinction. In addition, genetic variation at other loci may be greatly reduced during the purging period because of the reduction of the effective population size so that the future potential for adaptation in the population may be lost. In other words, the advantages of eliminating a large part of the genetic load in a short period of time may be outweighed either by the potential cost from a higher risk of immediate or long-term extinction or from increased future risk in loss of adaptive potential. My goal in this study is generally to determine under what conditions these effects are important and when they are not significant in the most extreme type of inbreeding in vertebrates, full-sib mating.

\section{Background}

To understand the process by which inbreeding depression may be reduced, the genetic basis and extent of inbreeding depression must first be understood. For inbreeding depression resulting from lowered viability in Drosophila, it appears that approximately half the effect is due to lethals and half is due to detrimental alleles of much smaller effects (Simmons \& Crow, 1977; Charlesworth \& Charlesworth 1987). The lethals appear to be nearly completely recessive while the detrimental alleles have a substantial effect in heterozygotes, that is, the detrimentals are not as recessive as the lethals (see details below).

The estimated number of lethal equivalents, that is the effective number of genes with lethal alleles (Crow \& Kimura, 1970; Cavalli-Sforza \& Bodmer, 1971), that reduce viability in endangered captive species appears to vary greatly among species. For example, it may be very low as in the Kudu, Bongo or several other species in which there appears to be very low inbreeding depression; or it can reach very high levels as in Wied's red-nosed rat which is estimated to have 15 lethal equivalents (Ralls et al., 1988). The basis for this large range of genetic load in different organisms is not known but it may be associated with past population sizes or past levels of inbreeding. There is some support for the view that the incidence of recessive genetic diseases is lower in some South Indian human populations that historically have had higher rates of consanguineous marriages (Sanghvi, 1966; however, see Bittles et al., 1991). We should note that inbreeding depression may also be expressed through other fitness components, such as male mating ability (Sharp, 1984; Miller \& Hedrick, 1993) or female fecundity (Marinkovic, 1967).

\section{Model}

Let us assume that the three genotypes at a biallelic locus, $A_{1} A_{1}, A_{1} A_{2}$ and $A_{2} A_{2}$, have relative viability values of $1,1-h s$ and $1-s$ where $s$ is the selective disadvantage of $A_{2} A_{2}$ and $h$ is the level of dominance. Further, the relative viability of an individual over all loci (I will be examining from two to 32 loci influencing viability) is the product of the individual locus viability values or $w=w_{\mathrm{A}} w_{\mathrm{B}} w_{\mathrm{C}} \ldots$, where $w$ is the overall viability of an individual, and, for example, $w_{\mathrm{A}}$ is the relative viability of the individual at the $A$ locus (this multiplicative model is often assumed for viability loci, e.g. Hedrick, 1985; Charlesworth \& Charlesworth, 1987). All the loci are assumed to assort independently, that is they are unlinked. It is also assumed that there is no mutation at these loci over the period of inbreeding.

In order to understand the effects of purging inbreeding depression, I will examine the influence of the most extreme type of inbreeding in vertebrates and dioecious organisms in general, continuous full-sib mating. The initial parent generation for each replicate 
is composed of two unrelated, diploid individuals. At every locus, one of the four initial alleles is randomly designated to have a selective disadvantage as a homozygote. The other three alleles have no disadvantage and are selectively equivalent. From this pair of individuals, a given number of zygotes is randomly generated (using uniform random numbers to mimic genetic segregation and independent assortment). In all the cases discussed below, the number of zygotes generated for each mating is 10 except where I explicitly examine the effect of progeny number. All replicate full-sib lines were followed for 20 generations and 1000 replicates were run for each combination of parameters.

Selection, based on the relative viability values of the individual zygotes, then occurs so that the number of surviving progeny may be less than the number of zygotes. If the viability of the individual is unity, then it survives to be a potential parent. If the viability is less than unity, a random number is generated and compared to the viability of the individual to determine if the individual survives (it survives if the uniform random number is between 0 and $w$ ). The relative survival in the progeny group is defined as the actual proportion of the zygotes that survive to adults and is equal to the mean fitness since there is no selection on other components of fitness. The number of lethal equivalents in an individual is equal to the sum of $s$, the selective disadvantage when homozygous, for all detrimental or lethal alleles (after Cavalli-Sforza \& Bodmer, 1971 , p. 364). The average number of lethal equivalents in a replicate divided by the initial number of lethal equivalents is the proportion of lethal equivalents remaining. Remember that because there is only one copy of the disadvantageous allele initially at all loci, it is in only one of the two initial individuals.

Two of the surviving progeny are chosen to be the parents of the next generation. If there are less than two surviving progeny, then the replicate becomes extinct. Further, if all the surviving progeny are of the same sex, then the replicate also becomes extinct. This occurs if a uniform random number is less than $(1 / 2)^{n-1}$ where $n$ is the number of surviving progeny. Because the progeny randomly selected to be the parents of the next generation are full-sibs, inbreeding rapidly accumulates over generations. Reduction in inbreeding depression within a replicate occurs because individuals with lethals or detrimental alleles have lower survival and thereby less chance to be selected as parents. Further, replicates that become fixed for these loci will have a higher probability of becoming extinct so that the remaining replicates are likely to have less genetic load.

\section{Results}

In order to comprehend the influence of the genetic basis of inbreeding depression on the rate of purging of lethal equivalents, average progeny survival and the probability of population extinction, I will examine various factors one by one. First, I will examine the influence of the number of lethal equivalents, the effect of spreading a given number of lethal equivalents over more loci, and the impact of different levels of dominance when all the loci are equivalent for the above parameters. Next, I will examine the situation that mimics the genetic load as estimated from the research in D. melanogaster in which I assume there are two different types of loci, lethals and detrimentals, with different levels of dominance. Finally, I will examine the effect of purging on extinction when the loci influence female fecundity or male mating ability.

\section{Different lethal equivalents}

First, let us examine the level of inbreeding depression, progeny survival (in the surviving lines), and the probability of extinction when there are different initial numbers of lethal equivalents, all due to recessive lethals. Figure 1 gives these values over 20 generations of full-sib mating (averaged over 1000 replicates) when there are initially $1,2,4$ or 8 lethal equivalents $(2,4,8$ or 16 recessive $(h=0)$, lethal $(s=1)$ loci). The proportion of lethal equivalents (Fig. 1a) drops very quickly for all levels of initial lethal equivalents so that by generations 5 and 10 only about 40 and 10 per cent, respectively, of the initial number of lethal equivalents remain. Obviously, inbreeding depression is quickly purged in this case.

On the other hand, progeny survival (Fig. 1b) and the probability of extinction (Fig. 1c) are quite different for the various initial numbers of lethals. The progeny survival shows a particularly interesting pattern, initially dropping drastically in the second generation and then recovering gradually to nearly 100 per cent by generation 15 . In the situation with eight initial lethal equivalents, the survival drops to only 46.6 per cent by generation 2, recovering to 84.6 per cent by generation 10 . When there is a high proportion of the lethal equivalents remaining, the progeny survival is low; but as the lethals are purged over time, the average survival value recovers.

Further, the probability of extinction varies with the number of lethal equivalents, ranging in generation 20 from only 10 per cent when there is one lethal equivalent to nearly 80 per cent when there are eight lethal equivalents (Fig. 1c). Nearly all the extinctions occur in the first five generations when the lethal alleles are first 

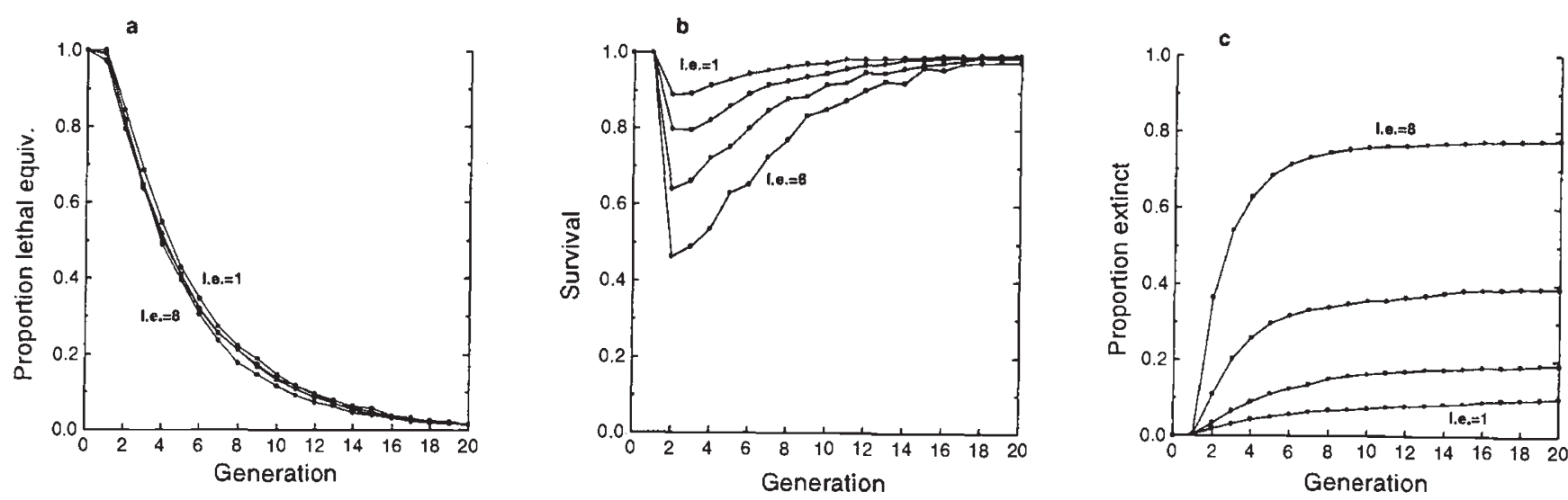

Fig. 1 The effect of continous full-sib mating when there are 1,2, 4 or 8 lethal equivalents (l.e.) because there are 2, 4,8 or 16 loci influencing viability; all the genetic load is in lethals $(s=1)$, and there is complete recessivity of these genes. The various parts of the figure give, over generations, (a) the proportion of lethal equivalents remaining, (b) the average individual survival and $(c)$ the cumulative proportion of the replicate populations that have become extinct.

being expressed as homozygotes as a result of the fast increase in the inbreeding coefficient. After this, the lethals have been purged, the progeny survival has recovered and, as a result, there are few extinctions except those due to not having individuals of both sexes. Assuming that there are 10 progeny in each generation, the probability of extinction over 20 generations due to the presence of only one sex is approximately $20(1 / 2)^{9}=3.9$ per cent. Of course, if there are fewer than 10 surviving progeny, then the rate of extinction due to the absence of one sex would be higher. Therefore, when there is one lethal equivalent, inbreeding depression causes a maximum increase of 10 per cent -3.9 per cent $=6.1$ per cent extinctions before the lethals are purged.

\section{Different selection coefficients}

Next, let us examine the influence of different selection coefficients for a given initial number of lethal equivalents, that is, the total genetic load is spread over greater numbers of loci. Figure 2 gives the values when one lethal equivalent is spread over $2,4,8,16$ or 32 loci and $s=1,0.5,0.25,0.125$ or 0.0625 , respectively, and there is complete recessivity, $h=0$. Notice in this case the loss of lethal equivalents (Fig. 2a) is progressively slower as the detrimental effect is spread over more and more loci. For example, with 32 loci after 20 generations, 82.2 per cent of the initial value of lethal equivalents are left, that is, very little purging has actually occurred.

This reduction in purging rate is due in part to the fixation of detrimental alleles. For example, after 20 generations when $s=0.0625,96.6$ per cent of the loci are fixed (data not shown in figures). Of these fixed loci, 20.7 per cent are fixed for alleles with a selective disadvantage. Since the initial frequency of each of these detrimental alleles was 0.25 , selection has reduced their overall frequency only a small amount while genetic drift has played a much more important role. In other words, the inbreeding depression is not purged as with lethals, but becomes fixed because the magnitude of selection is small relative to the effect of genetic drift. As a result, the survival values do not recover and, for example, with $s=0.0625$, they level off at a value below 0.7 (Fig. 2b). (If all initial alleles are equally likely to be fixed, genetic drift is much more important than selection so that the expected fitness when all loci are fixed in this case is $(1-0.0625)^{32 / 4}=$ 0.597 , slightly lower than the value observed, as is expected.) Consequently, the proportion of extinctions continues to increase over time so that for $s=0.0625$, 46.0 per cent of the populations have become extinct by generation 20 (Fig. 2c). Interestingly, this higher extinction rate is much the same when the genetic load is spread over loci with $s=0.25,0.125$ or 0.0625 . This suggests that the probability of extinction at any given generation for these conditions is relatively constant as the effect of individual loci becomes smaller.

\section{Different dominance levels}

Now let us examine the effect of various levels of dominance when there are lethals $(s=1)$ at four loci so that the number of initial lethal equivalents is two. In this case, the purging of the lethal equivalents occurs rapidly for all levels of dominance but is fastest for higher levels of dominance (Fig. 3a). For example, 

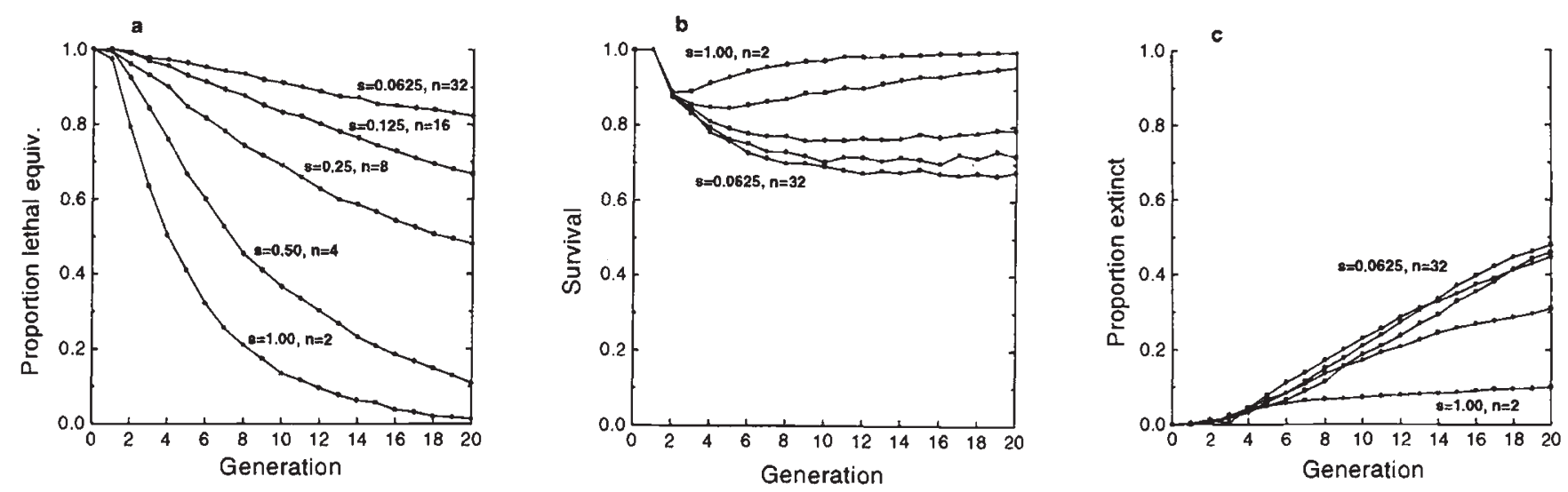

Fig. 2 The effect of continuous full-sib mating when there is one lethal equivalent and all the loci are completely recessive $(h=0)$. The genetic load is divided into different numbers of loci $(n)$ with following different selective effects: $s=1, n=2 ; s=0.5$, $n=4 ; s=0.25, n=8 ; s=0.125, n=16$; and $s=0.0625, n=32$. (a)-(c) are as in Fig. 1 .

when $h=0.5$, the proportion of lethal equivalents remaining after five generations is only 0.070 while when $h=0$, the proportion remaining is 0.428 . This difference occurs because selection acts against lethals when they are in heterozygotes if $h>0$.

The progeny survival when there is higher dominance has a different initial pattern from the case of complete recessivity (Fig. $3 b$ ). This occurs because the initial survival values are lower when $h>0$ and then increase over time as the lethals are purged. For all levels of dominance, the genetic load is essentially purged by generation 10 . The extinction of populations due to genetic causes occurs only in the first few generations for all levels of dominance (Fig. 3c). The highest extinction rate occurs with the higher levels of dominance with over 50 per cent of the populations becoming extinct in the first three generations when $h=0.5$.

The pattern is somewhat different when two lethal equivalents are spread out over 16 loci each with $s=0.25$. Figure 4 (a) shows the loss of lethal equivalents in this case for the five levels of dominance. If this is compared to Fig. 3(a), it is obvious that the rate of loss is slower for all levels of dominance. The survival of progeny, although quite different initially, becomes very similar for the different levels of dominance after about five generations (Fig. 4b). The survival then gradually increases to approximately 0.71 by generation 20 (at this point over 95 per cent of the loci are fixed in the extant lines, so the survival can only increase slightly after this). The extinction rate is much higher here with $s=0.25$ (Fig. 4c) than when $s=1$ (Fig. $3 c$ ). In fact, by generation 20 , between 75 and 90 per cent of the lines have become extinct.

\section{Genetic load due to both lethals and detrimentals}

As estimated by Simmons \& Crow (1977), the genetic load in Drosophila appears to be nearly equally divided between that due to lethals and that due to detrimentals. The lethals have been estimated to have nearly complete recessivity with an average $h=0.02$. On the other hand, the detrimentals are not so completely recessive with an average $h=0.2$ to 0.35 . To determine the effect of inbreeding depression based on both types of loci, I examined the case with two lethal equivalents in which either all the load is from lethals with $h=0.02$ (four loci), all the load is from detrimentals with $s=0.125$ and $h=0.25$ (16 loci), or half the load is from lethals with $h=0.02$ (two loci) and half from detrimentals with $s=0.125$ and $h=0.25$ (eight loci).

The results from these simulations are given in Fig. 5 in which the results labelled mixed are those with half of the genetic load from lethals and half from detrimentals. The results from this mixed source of genetic load are close to the average of the cases in which the load is exclusively due to lethals or to detrimentals. For both the proportion of lethal equivalents remaining and average survival, the results are somewhat closer to the findings when there are only detrimentals while for the proportion of populations that have gone extinct, the values for the mixed model are very close to the average. In this mixed case, the two types of loci behave much as they do when studied separately, that, is the lethals are quickly purged and some of the detrimentals are purged but a significant number are fixed, thereby lowering the overall viability. For example, there was 

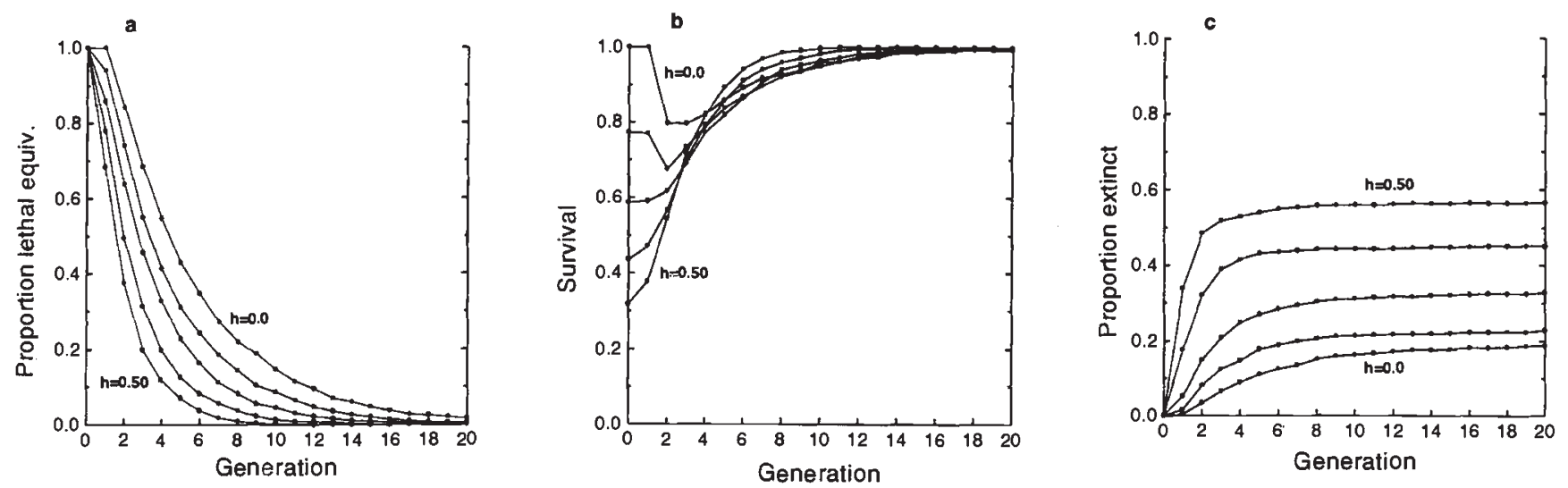

Fig. 3 The effect of continuous full-sib mating when there are two lethal equivalents that are the result of selection of lethals $(s=1)$ at four loci. The level of dominance $(h)$ is $0.0,0.125,0.25,0.375$ or 0.5 . (a)-(c) are as in Fig. 1.
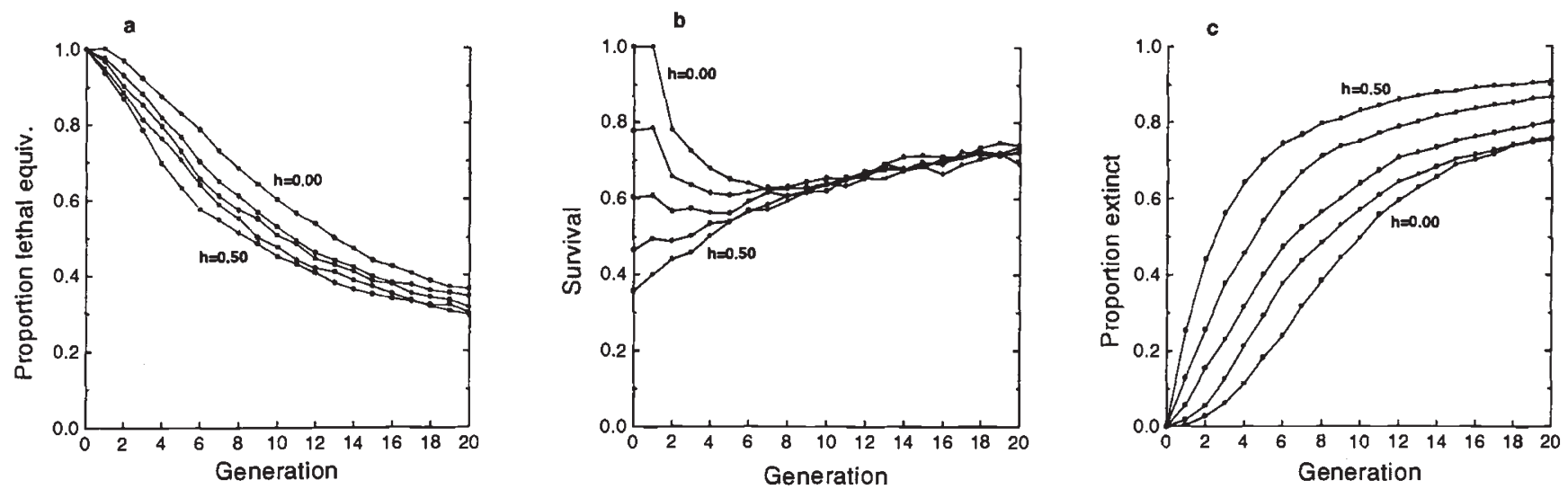

Fig. 4. The effect of continuous full-sib mating when there are two lethal equivalents that are the result of selection of detrimentals $(s=0.25)$ at 16 loci. The level of dominance $(h)$ is $0.0,0.125,0.25,0.375$ or 0.5 . (a)-(c) are as in Fig. 1.
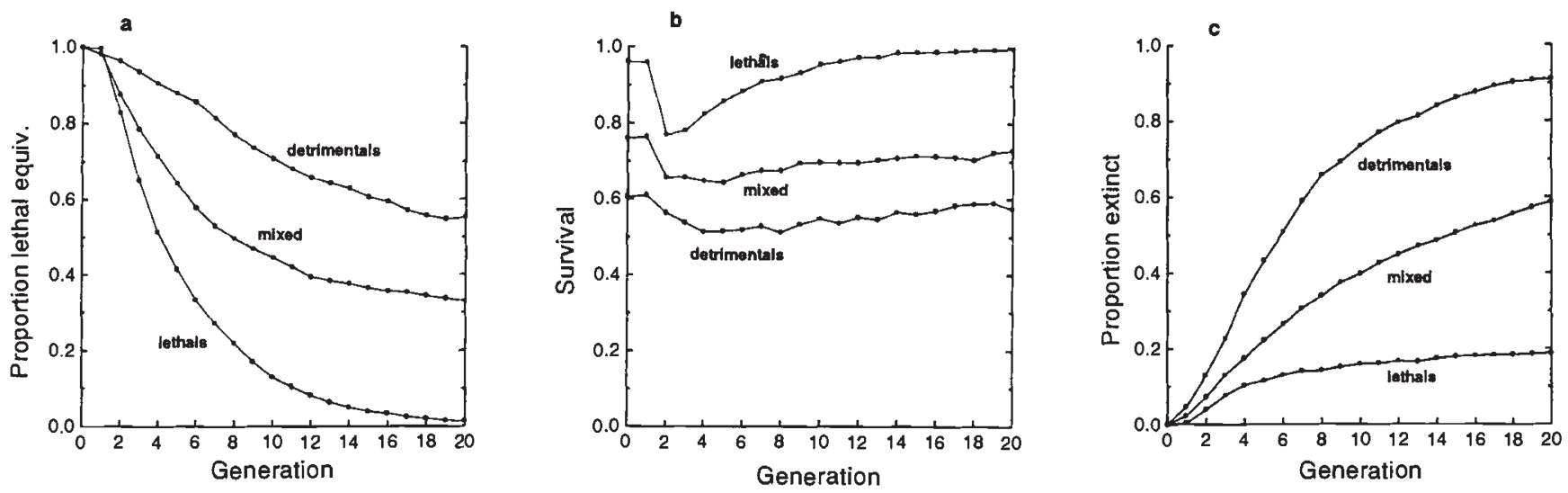

Fig. 5 The effect of continous full-sib mating when there are initially two lethal equivalents and either the load is due to lethals with $h=0.02$ (lethals), the load is due to detrimentals with $s=0.125$ and $h=0.25$ (detrimentals), or the load is due to an equal mix of these two types of loci (mixed). (a)-(c) are as in Fig. 1. 
no strong effect of the presence of lethals on the fixation of detrimentals. This is not unexpected because the loci in these simulations are unlinked and do not show epistasis.

\section{Male mating success and female fecundity}

Because other components of fitness are also affected by inbreeding, I also examined the effect of full-sib mating when the alleles reduced male mating success or female fecundity instead of viability. This was carried out by examining the genotype of one of the two individuals randomly chosen to be parents (because both male mating ability and female fecundity are by definition sex-limited and can only occur in one sex). Male mating ability or female fucundity were determined for the genotype of the chosen parent in the same manner as viability was determined above and this value was then used to generate progeny. For example, when examining female fecundity in which the relative fecundity of a given female is $w_{\mathrm{f}}$ (this value is bounded by unity and zero), an offspring is produced when a uniform random number falls between 0 and $w_{\mathrm{f}}$. Therefore, the expected number of progeny from this mating is $w_{\mathrm{f}}$ multiplied by the maximum number of progeny (which was again set to 10 in these simulations). This approach may overestimate the reduction of male mating ability if it is assumed that in an actual full-sib mating, a male would have multiple opportunities over several days to mate with its sibling.

Figure 6 gives these results when there is one, let us call it sterility equivalent, spread between $2,4,8,16$ or 32 loci with $h=0$. The reduction of sterility equivalents (Fig. 6a) occurs in much the same manner as when genes influence viability (Fig. $2 \mathrm{a}$ ). The relative productivity, which is defined as the number of progeny produced for a given female or male out of a given maximum number of progeny possible (Fig. $6 \mathrm{~b}$ ), also changes in much the same pattern except that the value for two loci with $s=1.0$ is always 1.0 . This results from the fact that the individual of concern (the female when it is female fecundity or the male when it is male mating success) is either normally fecund or mates normally or is not capable of laying eggs or mating. In this latter case, the line will go extinct because there will be no progeny. The extinction pattern for this case (Fig. 6c) is different from that for viability (Fig. 2c) in that the extinction rate is highest for the highest $s$ initially when there is fecundity or mating selection. This difference occurs because there is less opportunity for the population to cope with the initial load before it is purged since extinction results when only one individual parent fails to reproduce and not a whole progeny array.

\section{Number of progeny}

Obviously, Drosophila and a number of other dioecious organisms may have many more progeny than 10 while other organisms may have less than 10 offspring. What is the effect of having more than 10 progeny in the above simulations? First, if we carry out the same simulations as given in Figs 1-6 but with the number of progeny equal to 40 or 80 , the results are virtually identical to the results when there are 10 progeny for both lethal equivalents and survivorship. On the other hand, with higher progeny numbers, there is very little extinction for progeny numbers of 40 and virtually no extinction for runs with progeny numbers of 80 . In other words, when there are high progeny numbers, the genetic load is purged when there are lethals and no extinction results. When there are
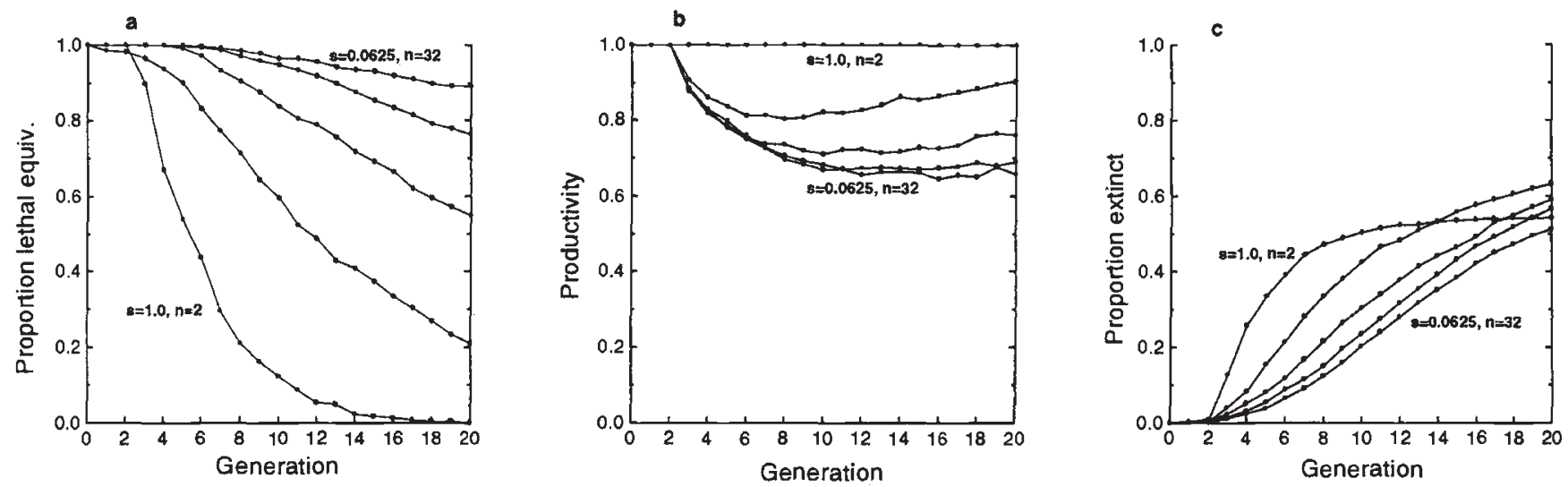

Fig. 6 The effect of continuous full-sib mating when there is one lethal equivalent affecting either female fecundity or male mating ability and $h=0$. The genetic load is spread over $2,4,8,16$ or 32 loci $(n)$. The various parts of the figure give, over generations, (a) the proportion of lethal equivalents remaining, (b) the relative number of progeny per parent and (c) the cumulative proportion of the replicate populations that become extinct. 
detrimentals, some of the load is fixed as with lower progeny numbers, which consequently lowers the fitness. However, extinction does not occur because the higher progeny number acts as a buffer against this reduced fitness. In other words, a higher reproductive capacity acts as a buffer against extinction during the period of low fitness when the genetic load is being purged.

Many large mammals produce fewer than 10 offspring per mating. If we carry out the same simulations as above when the number of progeny is five, the probability of extinction is very high over 20 generations and is dominated by extinction caused by lack of one of the sexes. The remaining populations generally have a slightly lower genetic load and survival than the values given in Figs 1-6, apparently due to a slightly higher extinction rate of the populations that have not purged their load. In a follow-up study, I will examine in more detail the effect of different progeny numbers, particularly in a population that is changing in size.

\section{Discussion}

The simulations in this study have demonstrated that the inbreeding depression in a population with recurrent full-sib mating may indeed be reduced or virtually eliminated in some situations in a captive breeding programme designed to purge genetic load through close inbreeding. However, two crucial findings for endangered species are that, first, the probability of extinction of the population may often be greatly increased during the period in which the inbreeding depression is being purged and second, the mean fitness may be reduced as a result of the purging attempt so that the probability of extinction in the long term is increased. More specifically, the genetic load due to lethals can be purged from a population if the number of lethals is not too large and the progeny size is sufficient to cope with the temporary low fitness experienced during the purging process. On the other hand, if the genetic load is due to detrimentals, they may become fixed during the period of attempted purging and thereby permanently lower the population fitness and lead to a higher probability of extinction. When the genetic load is due both to lethals and detrimentals as found in Drosophila, part of the genetic load may be purged while part of it may actually be fixed. These general results appear to hold as well for inbreeding depression influencing female fecundity or male mating ability.

One possible way to avoid fixation of detrimental alleles and the resulting increased probability of extinction is to find a rate of inbreeding that is high enough to eliminate the lethals but low enough so that the fixation of detrimentals is kept to a minimum. Of course this strategy would vary depending upon the constitution of the genetic load, both in terms of the effects of the loci and their level of dominance. In a follow-up study, using both larger population sizes and pedigrees from captive breeding programmes, I will examine if there is an optimum rate of increase of inbreeding, a rate that both eliminates load and maintains fitness without causing an undue increase in extinction.

As I stated in the description of the model, I have assumed that there is no mutation in the present simulations and have, therefore, examined the joint effects of selection, inbreeding and population extinction over a relatively short period, in evolutionary terms. This approach was chosen to mimic generally an endangered species, over its first few generations in captivity, that was founded with a small group of individuals from a larger population which was close to a mutation-selection balance. Barrett \& Charlesworth (1991) and D. Charlesworth (unpub. obs.) have actually examined the effects of self-fertilization on the genetic load in the presence of mutation and have reached general conclusions similar to those presented here. On the other hand, Lynch \& Gabriel (1990) suggest that very small populations over a period of time may have a decline in fitness due to the fixation of new, detrimental mutants, leading to what they term a 'mutational meltdown'. However, R. Lande (unpub. obs.) has shown that mutational meltdown is of much less importance in influencing extinction than are other factors.

In addition, I have assumed multiplicative fitness values over loci. If there are epistatic interactions such that the detrimental alleles reduce fitness more than the multiplicative model when there are multiple genes (as suggested by Kimura \& Maruyama, 1966; Charlesworth et al., 1992), then fitness may be lowered more than in the above simulations and the probability of extinction raised.

Empirical studies of continuous full-sib mating have shown quite high values of extinction, varying from 100 per cent extinction in Japanese quail over four generations (Sittman et al., 1966) and 95 per cent extinction over 20 generations in house mice (Bowman \& Falconer, 1960) to around 50 to 75 per cent in several Drosophila experiments (R. Frankham, unpubl. obs.). Generally, it has been suggested that the high extinction rate observed in many organisms upon inbreeding is the result of a high initial genetic load. In light of the findings presented above, we should be able to determine the type of initial genetic load responsible for the high extinction rate. For example, if an initial genetic load of the amount normally suggested for Drosophila and mice, around two to four lethal equivalents, was due principally to lethals, it should not result in this high rate of extinction because the progeny 
number is high enough to avoid extinction while the lethals are being purged. On the other hand, if the load is entirely due to detrimentals, extinction should occur over an extended period of time rather than be concentrated in a single, early period. However, when fecundity and male mating are influenced by lethals, the effect should be concentrated in the early generations, suggesting that these variants may be implicated in causing the observed extinctions. Of course, in addition to the extinctions resulting from genetic causes, a background extinction rate due to environmental or unknown causes may be important and may somewhat obscure the genetic basis of extinctions.

It has been a concern that a programme to purge a population of lethal equivalents would reduce the extent of variations at other loci (Hedrick \& Miller, 1992). The extent of this effect may be determined for neutral loci by calculating the influence of the particular breeding programme on the inbreeding coefficient in the population and assuming no associations among loci (if there are significant associations among loci generated by inbreeding and selection, then the rate of increase in inbreeding may be different from that for neutral genes). For example, in the case simulated here, the inbreeding coefficient for continous full-sib mating can be used to calculate the expected reduction in the amount of heterozygosity for neutral loci. Even though a few generations of full-sib mating may purge lethals fairly effectively, as shown in Fig. 1, as a result, a much reduced proportion of the genetic variation at other loci may be left for future adaptive response after only a short period.

Templeton \& Read (1984) concluded in their evaluation of the breeding programme they devised for Speke's gazelle that 'inbreeding depression can be rapidly and effectively reduced by an appropriate breeding program, hence an inbreeding depression does not constitute an insurmountable barrier to the long-term maintenance of a species in which inbreeding cannot be avoided'. On the other hand, Barrett \& Charlesworth (1991) conclude that a programme to purge inbreeding depression 'could decrease fitness considerably, with little recovery under inbreeding'. Part of this difference in opinion may be that the rate and extent of inbreeding in the Speke's gazelle captive population reported by Templeton \& Read (1984) was much lower than in the study of the annual water hyacinth by Barrett \& Charlesworth (1991). The two groups of gazelles compared by Templeton \& Read (1983) had average inbreeding coefficients of 0.128 before and 0.149 after the inbreeding episode which took place between 1979 and 1983 (two to three generations) (A. Templeton, pers. comm.). Obviously, this increase of 0.021 in the inbreeding coefficient is much less than that resulting from one generation of full-sib mating and much more than an order of magnitude less than the five generations of self-fertilization in the experiment of Barrett \& Charlesworth (1991).

Templeton \& Read $(1983,1984)$ estimated that the number of lethal equivalents for 30-day survival was reduced from about six to about three, an approximately 50 per cent reduction, as a result of their breeding programme. Because the inbreeding coefficient was increased only 0.021 during this period, it appears that either the variety of genetic scenarios that I examined did not include this particular situation or that they had an unusually fortunate reduction in lethal equivalents. For example, the near maximum reduction in lethal equivalents with an increase of inbreeding coefficients of 0.25 was only about 20 per cent in my simulations when the load was due entirely to lethals (Fig. 1).

Templeton \& Read $(1983,1984)$ suggest that as inbreeding is increased, several other traditional procedures also be employed, that is that the population be increased from its small founder number as fast as possible and attempts be made to equalize founder representation. In addition, Templeton \& Read (1983) suggest that additional artificial selection be used by choosing 'inbred but healthy animals' to be used as parents. It is possible that some of these procedures could result in purging of inbreeding depression while reducing the general rate of increase in inbreeding more than if randomly surviving individuals were chosen as parents as in my simulations. It would be necessary to examine the Speke's gazelle breeding programe and pedigree in detail to judge the potential importance of these factors. It is not intuitively clear how the number of lethal equivalents could be halved without exposing a substantial part of the genetic load to selection as homozygotes, a situation that seems unlikely given the small increase in the inbreeding coefficient observed in the gazelles over this period.

Although I have only examined purging of inbreeding depression and extinction under very close inbreeding here, it appears from these results and reflections that intentional purging of the inbreeding depression generally may not be a good strategy for captive breeding because the risk of extinction may be greatly increased. To determine if extinction is also likely in captive breeding situations in which the rate of increase of inbreeding is slower than here, I have undertaken a further study of the same phenomena in small populations and in populations mimicking captive pedigrees. It has been suggested (Lande \& Barrowclough, 1987) that mild inbreeding, for example a low frequency of full-sib mating in large populations, could purge most of the recessive lethals with little risk of extinction. In fact, there is some empirical support that slower rates of inbreeding can lead to more effec- 
tive purging of inbreeding depression (Ehiobu et al., 1989).

Other insight into the impact of inbreeding could be obtained by examining captive animal populations that have extensive pedigree information to determine whether any decrease in inbreeding depression, for example as in an increased juvenile viability, has occurred as the inbreeding coefficient has increased. Further studies to determine the genetic basis of load and extinction in inbred lines or other small populations might also give insight into the genetic changes which are of greatest potential concern when trying to maintain a population. Without this type of information, it appears that breeding strategies to maintain heterozygosity, avoid inbreeding or equalize founder contribution (e.g. Hedrick \& Miller, 1992) would be preferable to one based on purging inbreeding depression, because of the increased probability of extinction that may result.

\section{Acknowledgements}

I appreciate the constructive comments of Deborah Charlesworth, Dick Frankham, Bob Lacy, Mike Lynch, Phil Miller and several anonymous reviewers on an earlier draft of the manuscript. This research was supported by NSF BRF-8923007.

\section{References}

BARRETT, S. C. H. AND CHARLESWORTH D. 1991. Effects of a change in the level of inbreeding on the genetic load. Nature, 352, 522-524.

BITTLES, A. H., MASON, W. M., GREENE, J. AND RAO, N. A. 1991. Reproductive behavior and health in consanguineous marriages. Science, 252, 789-794.

BOWMAN, J. C. AND FALCONER, D. S. 1960. Inbreeding depression and heterosis of litter size in mice. Genet. Res., 1, 262-274.

BRown, A. H. D. 1979. Enzyme polymorphism in plant populations. Theor. Pop. Biol. 15, 1-42.

CAVALLI-SFORZA, L. L. AND BODMER, w. F. 1971. The Genetics of Human Populations. W. H. Freeman, San Francisco.

CHARLESWORTH, D. AND CHARLESWORTH, B. 1987. Inbreeding depression and its evolutionary consequences. Ann. Rev. Ecol.Syst., 18, 237-268.

CHARLESWORTH, D., MORGAN, M. T. AND CHARLESWORTH, B. 1992. The effect of linkage and population size on inbreeding depression due to mutational load. Genet. Res., 59, 49-61.

CROW, J. F. AND KIMURA, M. 1970. An Introduction to Population Genetics Theory. Harper and Row, New York.

DARWIN, C. R. 1868. Variation of Animals and Plants under Domestication. John Murray, London.

DARWIN, C. R. 1876. The Effects of Cross and Self Fertilization in the Vegetable Kingdom. John Murray, London.

EHIOBU, N. G., GODDARD, M. E. AND TAYLOR, J. F. 1989. Effect of rate of inbreeding on inbreeding depression in Drosophila melanogaster. Theor. Appl. Genet., 77, 123-137.
FRANKLIN, I. R. 1980. Evolutionary changes in small populations. In: Soulé, M. E. and Wilcox, B. A. (eds) Conservation Biology, pp. 135-149. Sinauer, Sunderland, MA.

HALDANE, J. B. S. 1937. Some theoretical results of continued brother-sister mating. J. Genet., 34, 265-274.

HEDRICK, P. W. 1985. Genetics of Populations. Jones and Bartlett, Boston.

HEDRICK, P. W. 1990. Mating systems and evolutionary genetics. In: Wohrmann, K. and Jain, S. (eds) Population Biology: Ecological and Evolutionary Viewpoints, pp. 83-114. Springer, New York.

HEDRICK, P. W. AND MILLER, P. S. 1992. Conservation genetics: techniques and fundamentals. Ecol. Appl., 2, 30-46.

KIMURA, M. AND MARUYAMA, T. 1966. The mutational load with epistatic gene interactions in fitness. Genetics, 54, 1337-1351.

LACY, R. C. 1992. The effects of inbreeding on isolated populations: are minimum viable population sizes predictable? In: Fiedler, P. L and Jain, S. K. (eds) Conservation Biology: the Theory and Practice of Nature Conservation, Preservation and Management, pp. 277-296. Chapman and Hall, New York.

LANDE, R. AND BARROWCLOUGH, G. F. 1987. Effective population size, genetic variation, and their use in population management. In: Soulé, M. E. (ed.) Viable Populations for Conservation, pp. 87-123. Cambridge University Press, Cambridge.

LERnER, I. M. 1954. Genetic Homeostasis. Oliver and Boyd, Edinburgh.

LYNCH, M. AND GABRIEL, w. 1990. Mutation load and the survival of small populations. Evolution, 44, 1725-1737.

MARINKOVIC, D. 1967. Genetic loads affecting fecundity in natural populations of $D$. pseudoobscura. Genetics, 56, 61-71.

MILLER, P. S. AND HEDRICK, P. W. 1993. Inbreeding and fitness in captive populations: lessons from Drosophila. Zoo Biol., 12, 333-352.

RALLS, K., BALLOU, J. AND TEMPLETON, A. 1988. Estimates of lethal equivalents and the cost of inbreeding in mammals. Conserv. Biol., 2, 185-193.

REEVE, E. C. R. 1955. Inbreeding with homozygotes at a disadvantage. Ann. Rev. Genet., 21, 277-288.

SANGHVI, L. D. 1966. Inbreeding in India. Eugen. Quart., 13, 291-301.

SHARP, P. M. 1984. The effect of inbreeding on competitive male-mating ability in Drosophila melanogaster. Genetics, 106, 601-612.

SIMMONS, M. J. AND CROW, J. F. 1977. Mutations affecting fitness in Drosophila populations. Ann. Rev. Genet., 11, 49-78.

SITTMAN, K., ABPLANALP, H. A. AND FISHER, R. A. 1966. Inbreeding depression in Japanese quail. Genetics, 54, 371-379.

TEMPLETON, A. R. AND READ, B. 1983. The elimination of inbreeding depression in a captive herd of Speke's gazelle. In: Schonewald, C. M., Chambers, S. M., MacBryde, B. and Thomas, L. (eds) Genetics and Conservation, pp. 241-262. Benjamin/Cummings, Menlo Park, CA.

TEMPLETON, A. R. AND READ, B. 1984. Factors eliminating inbreeding depression in a captive herd of Speke's gazelle. Zoo Biol., 3, 177-199.

WRIGHT, s. 1977. Evolution and Genetics of Populations, vol. 3. Experimental and Evolutionary Deductions. University of Chicago Press, Chicago. 